\title{
$\mathrm{Nd}$ 掺杂硅酸钙及其复合电纺丝膜的制备及性能研究
}

\author{
马玲玲 1,2 , 常 江 1,2 \\ (1. 中国科学院上海砫酸盐研究所高性能陶瓷与超微结构国家重点实验室, 上海 200050; 2. 中国科学院大学材料与光电 \\ 研究中心，北京 100049)
}

摘 要: 同时具有光热效应与组织修复活性的生物材料在再生医学领域具有潜在应用前景。考虑到稀土元素 $\mathrm{Nd}$ 的荧 光发光与光热双重特性, 结合钙硅基 ( $\mathrm{Ca}-\mathrm{Si}$ ) 生物材料优异的组织修复活性, 有可能制备出多功能组织组织损伤 修复材料。本研究通过在硅酸钙中引入 $\mathrm{Nd}$ 元素, 采用共沉淀方法及 $800 \mathrm{C}^{\circ}$ 以上煅烧温度成功制备了 $\mathrm{Nd}-\mathrm{Ca}-\mathrm{Si}$ 基 生物陶瓷粉体 $(\mathrm{Nd} / \mathrm{CS})$, 并评价了粉体的物相组成、光热和苂光性能; 进一步采用静电纺丝技术制备了陶瓷粉体 /高分子复合膜, 并评价了其理化性能与生物学性能。结果显示, Nd/CS 陶瓷粉体和复合膜不但具有优异的光热性 能与荧光性能, 还具有较好的荧光测温性能。细胞实验证实复合膜具有优良的生物学性能。这种集光热治疗、荧光 成像/荧光测温、生物活性功能于一体的 Nd-Ca-Si 基生物陶瓷及其复合材料有望应用于再生医学领域。

关 键 词:钙硅基; 稀土掺杂; 生物学性能; 静电纺丝

中图分类号: TQ174 文献标识码: A

\section{Nd-doped Calcium Silicate: Photothermal Effect, Fluorescence Performance, and Biological Properties of Its Composite Electrospun Membrane}

\author{
MA Lingling ${ }^{1,2}$, CHANG Jiang ${ }^{1,2}$ \\ (1. State Key Lab of High Performance Ceramics and Superfine Microstructure, Shanghai Institute of Ceramics, Chinese Acad- \\ emy of Sciences, Shanghai 200050, China; 2. Center of Materials Science and Optoelectronics Engineering, University of Chi- \\ nese Academy of Sciences, Beijing 100049, China)
}

\begin{abstract}
Biomaterials with both photothermal effect and tissue repair activity have potential applications in the field of regenerative medicine. Considering the dual functions of fluorescence and photothermal effect of rare earth element $\mathrm{Nd}$, and the excellent tissue repair biological activity of calcium-silicon $(\mathrm{Ca}-\mathrm{Si})$ based materials, here we successfully prepared Nd-Ca-Si-based bioceramics (Nd/CS) powder by introducing Nd into calcium silicate through co-precipitation method and sintering at temperatures above $800^{\circ} \mathrm{C}$, and evaluated phase composition, photothermal, and fluorescence properties of the powder. For application in soft tissue repair, we further prepared a ceramic powder/polymer composite electrospun membrane, and evaluated its physical, chemical and biological properties. The results showed that Nd/CS ceramic powder and composite membrane not only had excellent photothermal performance and fluorescence emitting effects, but also showed fluorescence thermometry property. Preliminary cell experiments confirmed that the composite membrane was bioactive to stimulate fibroblast activity. This Nd-Ca-Si-based bioceramic and its composite material with integrated photothermal, fluorescence imaging/fluorescence thermometry properties, and bioactivity, may have potential applications in regenerative medicine.
\end{abstract}

Key words: Calcium silicon base; rare earth doping; biological activity; electrospinning

同时具有生物活性与光热效应如光热催化 ${ }^{[1] 、}$

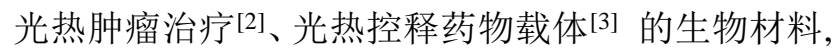

收稿日期: 2020-12-14; 收到修改稿日期：2021-01-07

基金项目：国家重点研发计划（2016YFC1100200） National Key Research and Development Plan of China (2016YFC1100201)

作者简介：马玲玲（1994-), 女, 博士研究生. E-mail: malingling@mail.sic.ac.cn MA Lingling (1994-), female, PhD candidate. E-mail: malingling@mail.sic.ac.cn

通讯联系人: 常 江, 研究员. E-mail: jchang@mail.sic.ac.cn

CHANG Jiang, professor. E-mail: jchang@mail.sic.ac.cn 
在生物医学、催化等领域展示了巨大应用前景。但 一般光热材料的光热剂在受到单一激发光光源的激 发下, 只能将光能转化为热能, 通过热作用达到特 定的应用目的 ${ }^{[4]}$ 。对光热温度的测量也仅限于采用 红外热像仪测量物体的表面温度, 无法准确测量材 料所处位置的实际温度, 更无法探测材料在生物体 内的降解等实际存在状态。目前, 在光热治疗过程 中兼顾温度监测的研究大都集中在纳米材料领域 ${ }^{[5-}$ 7]。但纳米材料缺少生物活性, 有潜在毒性, 且靶向 性仍有不足 ${ }^{[8-9]}$, 限制了其临床应用。因此, 制备一 种既具有温度监测/生物成像功能, 又具有组织再生 活性的多功能块体光热材料具有重要意义。

与其他有机、无机、金属等光热剂相比, 镧系 稀土元素钕 $(\mathrm{Nd})$ 具有独特的性能 ${ }^{[6,10]}$ 。 $\mathrm{Nd}$ 离子在 近红外 $808 \mathrm{~nm}$ 的激光光照下, 不仅能释放出热能, 还能释放出光能。利用 $\mathrm{Nd}$ 离子受激发后释放的热 能, 可以较容易地达到光热治疗所需的温度。由于 $\mathrm{Nd}$ 离子受激发后可释放光能, $\mathrm{Nd}$ 复合材料还具有 苂光温度监测、苂光成像等潜在应用 ${ }^{[6,11-12]}$ 。组织 再生材料研究发现, 钙硅基 ( $\mathrm{Ca}-\mathrm{Si}$ ) 生物材料如硅 酸钙 $\left(\mathrm{CaSiO}_{3}\right)$ 具有良好的生物活性, 其释放出的 $\mathrm{Ca} 、 \mathrm{Si}$ 离子具有刺激细胞增殖、粘附、迁移、成血 管基因表达的作用, 是一种优异的组织修复材料 [1315]。Ca-Si 基材料对骨等硬组织缺损、皮肤等软组织 创伤都有显著的修复作用 ${ }^{[16-17]}$ 。但至今尚未见将 $\mathrm{Nd}$ 离子受激发后的放热性能与钲硅基生物材料的生物 活性结合、综合发挥二者优势的研究报道。

本研究提出将 $\mathrm{Nd}$ 元素引入到钙硅基材料体系 中, 制备集光热、苂光发光与组织修复活性功能于 一体的生物材料。考虑到硬组织与软组织修复治疗 以及临床应用时对材料力学特性的不同要求, 本研 究进一步将 $\mathrm{Nd}-\mathrm{Ca}-\mathrm{Si}$ 基陶瓷粉体 ( $\mathrm{Nd} / \mathrm{CS}$ ) 均匀分 散在聚已内酯 (PCL) 中, 制备出陶瓷粉体/高分子 复合静电纺丝膜。系统评价了 $\mathrm{Nd}-\mathrm{Ca}-\mathrm{Si}$ 基生物陶瓷 粉体及静电纺丝复合膜的光热、苂光发光性能, 并 采用细胞实验评价了复合膜的生物活性。

\section{1 实验方法}

\section{1 实验药品}

本研究所用的药品四水合硝酸钘 $\left(\mathrm{Ca}\left(\mathrm{NO}_{3}\right)_{2} \bullet 4 \mathrm{H}_{2} \mathrm{O}\right)$ 、九水硅酸钠 $\left(\mathrm{Na}_{2} \mathrm{SiO}_{3}-9 \mathrm{H}_{2} \mathrm{O}\right)$ 、 氨水 $\left(\mathrm{NH}_{3} \cdot \mathrm{H}_{2} \mathrm{O}\right)$ 均购自中国医药集团上海化学试 剂有限公司。六水合硝酸钕 $\left(\mathrm{Nd}\left(\mathrm{NO}_{3}\right)_{3} \cdot 6 \mathrm{H}_{2} \mathrm{O}\right)$, 购
自阿拉丁试剂 (上海) 有限公司。聚已内酯 (PCL, $\mathrm{Mw}=80000$ ), 购自西格玛奥德里奇（上海）贸易有 限公司。1,1,1,3,3,3-六氟-2-丙醇, 购自九鼎化学 (上 海）科技有限公司。上述试剂均为分析纯。

\section{2 材料合成及 $\mathrm{Ca} 、 \mathrm{Si}$ 离子释放}

采用化学共沉淀法制备 $\mathrm{Nd}-\mathrm{Ca}-\mathrm{Si}$ 基生物活性 陶瓷粉体。具体操作如下: 将 $21.2523 \mathrm{~g}$ $\mathrm{Ca}\left(\mathrm{NO}_{3}\right)_{2} \cdot 4 \mathrm{H}_{2} \mathrm{O}$ 与 $4.2825 \mathrm{~g} \mathrm{Nd}\left(\mathrm{NO}_{3}\right)_{3} \cdot 6 \mathrm{H}_{2} \mathrm{O}$ 加入 200 $\mathrm{mL}$ 去离子水中, 磁力搅拌下溶解。然后用 $\mathrm{NH}_{3} \cdot \mathrm{H}_{2} \mathrm{O}$ 调节溶液 $\mathrm{pH}$ 值至 13 。再将 $56.84 \mathrm{~g}$ 的 $\mathrm{Na}_{2} \mathrm{SiO}_{3} \cdot 9 \mathrm{H}_{2} \mathrm{O}$ 溶解在 $400 \mathrm{~mL}$ 去离子水中, 并逐滴加入到上述硝 酸盐混合溶液中。最后将上述混合物在室温下磁力 搅拌 $12 \mathrm{~h}$ 后, 过滤, 分别用去离子水、无水乙醇洗 涤三遍, 放入 $60^{\circ} \mathrm{C}$ 烘箱干燥 $48 \mathrm{~h}$, 得到 $\mathrm{Nd}-\mathrm{Ca}-\mathrm{Si}$ 基 陶瓷粉体前驱体。随后将前驱体在 800、900、1000 ${ }^{\circ} \mathrm{C}$ 下炦烧 $3 \mathrm{~h}$, 用行星球磨机球磨 $10 \mathrm{~min}$, 过孔径为 $75 \mu \mathrm{m}$ 的篎。将制备的陶瓷粉体记为 $\mathrm{Nd} / \mathrm{CS}$ 。

采用静电纺丝技术制备 Nd/CS-PCL 复合膜。将 $1 \mathrm{~g}$ 的 PCL 与 $0 、 0.05$ 和 $0.1 \mathrm{~g}$ 的 $\mathrm{Nd} / \mathrm{CS}$ 生物陶瓷粉 体加入 $10 \mathrm{~mL}$ 的 1,1,1,3,3,3-六氟-2-丙醇中, 磁力摚 拌 $12 \mathrm{~h}$ 充分混匀。将混合液装入 $10 \mathrm{~mL}$ 注射器中, 以 $0.02 \mathrm{~mL} / \mathrm{min}$ 流速、8 10 KV 电压、 $15 \mathrm{~cm}$ 固定接 受距离作静电纺丝。将制备的样品分别记为 PCL、 Nd/CS-PCL5、Nd/CS-PCL10。

使用直径为 $13 \mathrm{~mm}$ 的打孔器将静电纺丝复合膜 制备成直径为 $13 \mathrm{~mm}$ 的圆形薄膜。将静电纺丝复合 膜圆片放入 PBS 中, 然后放入 $37{ }^{\circ} \mathrm{C}$ 恒温摇床, 在 1、3、5 和 $7 \mathrm{~d}$ 取上清液, 测试溶液 $\mathrm{Ca} 、 \mathrm{Si}$ 离子浓 度。

\section{3 材料的性能表征}

采用 XRD (Rigaku D/Max-2550 V) 分析 Nd/CS 生物陶瓷粉体的物相组成; 采用红外热像仪对 $\mathrm{Nd} / \mathrm{CS}$ 粉体和 Nd/CS-PCL 静电纺丝复合膜的光热 性能进行表征; 采用低温吸收光谱仪（FLS-980）表 征 $\mathrm{Nd} / \mathrm{CS}$ 粉体与 $\mathrm{Nd} / \mathrm{CS}-\mathrm{PCL}$ 静电纺丝复合膜的荧 光性能; 采用场发射扫描电子显微镜 (S-4800) 观 察静电纺丝复合膜的微观形貌; 采用电感耦合等离 子体发射光谱仪 (ICP-OES) 检测静电纺丝复合膜释 放出的 $\mathrm{Ca} 、 \mathrm{Si}$ 离子浓度。

\section{4 细胞迁移}

采用人源皮肤成纤维细胞 (HDF) 探究 Nd/CSPCL 静电纺丝复合膜对 HDF 细胞迁移的影响。

a) 将复合静电纺丝膜制备成直径为 $20 \mathrm{~mm}$ 的 圆形薄膜, 随后放入 6 孔的细胞培养板中, 用钛环 
固定。最后放入紫外交联仪中紫外灭菌 $4 \mathrm{~h}$ 。

b) 采用划痕实验法研究 HDF 细胞迁移: 选用 6 孔细胞培养板, 每孔接种 10 万个细胞, 放入 $37{ }^{\circ} \mathrm{C}$ 、 $5 \% \mathrm{CO}_{2}$ 的恒温细胞培养箱培养。待孔板内细胞密 度达到 $80 \%$ 左右时, 用 $1 \mathrm{~mL}$ 的枪头在孔板底部划 线, 然后在光学显微镜下观察划痕状态并采集图像。 加入灭菌后的圆形静电纺丝复合膜, 培养液中血清 浓度改为 $1 \%$, 继续培养 $12 \mathrm{~h}$ 后, 移除培养液、静 电纺丝复合膜, 采用 $4 \%$ 多聚甲醛溶液固定细胞、 $1 \%$ 的结晶紫溶液染色细胞 $5 \mathrm{~min}$, 用 PBS 润洗 3 次。 最后在显微镜下观察 HDF 细胞的形貌, 并采集图 像。

\section{5 细胞粘附}

本实验选用 HDF 细胞研究 Nd/CS-PCL 静电纺 丝复合膜对 HDF 细胞粘附的影响。

a）采用直径为 $13 \mathrm{~mm}$ 的打孔器将静电纺丝复 合膜制备成直径为 $13 \mathrm{~mm}$ 的圆形薄膜, 然后放入 24 孔的细胞培养板中, 用钛环固定。最后放入紫外交 联仪中紫外灭菌 $4 \mathrm{~h}$ 。

b) 在灭菌后的静电纺丝复合膜上种植 HDF 细 胞, 每孔种 5000 个 $\mathrm{HDF}$ 细胞。在 $37{ }^{\circ} \mathrm{C} 、 5 \% \mathrm{CO}_{2}$ 浓度的恒温细胞培养箱中培养 $24 \mathrm{~h}$ 后, 选用 $4 \%$ 的 多聚甲醛溶液固定细胞, PBS 润洗 3 次后, 采用梯 度浓度酒精进行脱水, 然后放入通风梪中过夜干燥。 最后使用场发射扫描电子显微镜（S-4800）观察静 电纺丝复合膜上粘附的 HDF 细胞形貌。

\section{2 结果与讨论}

\subsection{Nd/CS 的物相组成与光热性能}

不同烧结温度下 $\mathrm{Nd} / \mathrm{CS}$ 陶瓷粉体的物相组成如 图 1(a)所示, $700^{\circ} \mathrm{C}$ 烧结时 $\mathrm{Nd} / \mathrm{CS}$ 粉体为无定型态 的玻璃相。烧结温度升高, 至 $800^{\circ} \mathrm{C}$ 时, $\mathrm{Nd} / \mathrm{CS}$ 粉体 为结晶相。且 $800 \sim 1000{ }^{\circ} \mathrm{C}$ 烧结时, $\mathrm{Nd} / \mathrm{CS}$ 陶瓷粉 体的主晶相为硅酸钙 $\left(\mathrm{CaSiO}_{3}\right)$ 且有少量的二氧化 硅 $\left(\mathrm{SiO}_{2}\right)$ 相。 $800{ }^{\circ} \mathrm{C}$ 时, $\mathrm{Nd}$ 离子主要以富钕磷灰 石相 $\left(\mathrm{Ca}_{2} \mathrm{Nd}_{8}\left(\mathrm{SiO}_{4}\right)_{6} \mathrm{O}_{2}\right)$ 存在, 而 $900{ }^{\circ} \mathrm{C}$ 与 $1000{ }^{\circ} \mathrm{C}$ 时, $\mathrm{Nd}$ 离子主要以硅酸钕 $\left(\mathrm{Nd}_{2} \mathrm{Si}_{2} \mathrm{O}_{7}\right)$ 相存在。 $\mathrm{Nd} / \mathrm{CS}$ 粉体中 $\mathrm{Nd}$ 离子的存在形式直接受烧结温度的影响。 对不同温度烧结后的 $\mathrm{Nd} / \mathrm{CS}$ 粉体进行光热性能表 征 (图 1(b)) 发现, 随着烧结温度的升高, 粉体的 光热性能随之减弱。 $800{ }^{\circ} \mathrm{C}$ 烧结后的粉体光热性能 最好, $900{ }^{\circ} \mathrm{C}$ 次之, $1000{ }^{\circ} \mathrm{C}$ 烧结后粉体光热性能最 差。这些结果说明, 陶瓷粉体的相组成对其光热性
能有直接影响。 $\mathrm{Ca}_{2} \mathrm{Nd}_{8}\left(\mathrm{SiO}_{4}\right)_{6} \mathrm{O}_{2}$ 的光热性能高于 $\mathrm{Nd}_{2} \mathrm{Si}_{2} \mathrm{O}_{7}$, 这可能是由于 $\mathrm{Ca}_{2} \mathrm{Nd}_{8}\left(\mathrm{SiO}_{4}\right)_{6} \mathrm{O}_{2}$ 为六方晶 系 (空间群为: $\mathrm{P} 63 / \mathrm{m}(176)$, 晶胞参数为 $0.9532 \mathrm{~nm}$ $\times 0.9532 \mathrm{~nm} \times 0.7021 \mathrm{~nm})$, 而 $\mathrm{Nd}_{2} \mathrm{Si}_{2} \mathrm{O}_{7}$ 为四方晶系 (空间群为: $\mathrm{P} 141(76)$, 晶胞参数为 $0.6741 \mathrm{~nm} \times$ $0.6741 \mathrm{~nm} \times 2.452 \mathrm{~nm}$ ), 所以受到激光照射后, $\mathrm{Ca}_{2} \mathrm{Nd}_{8}\left(\mathrm{SiO}_{4}\right)_{6} \mathrm{O}_{2}$ 中的 $\mathrm{Nd}$ 离子相对较容易发生跃迁 进而释放光能与热能。考虑到材料光热性能的高低, 本实验选择 $800{ }^{\circ} \mathrm{C}$ 烧结的陶瓷粉体进行实验。
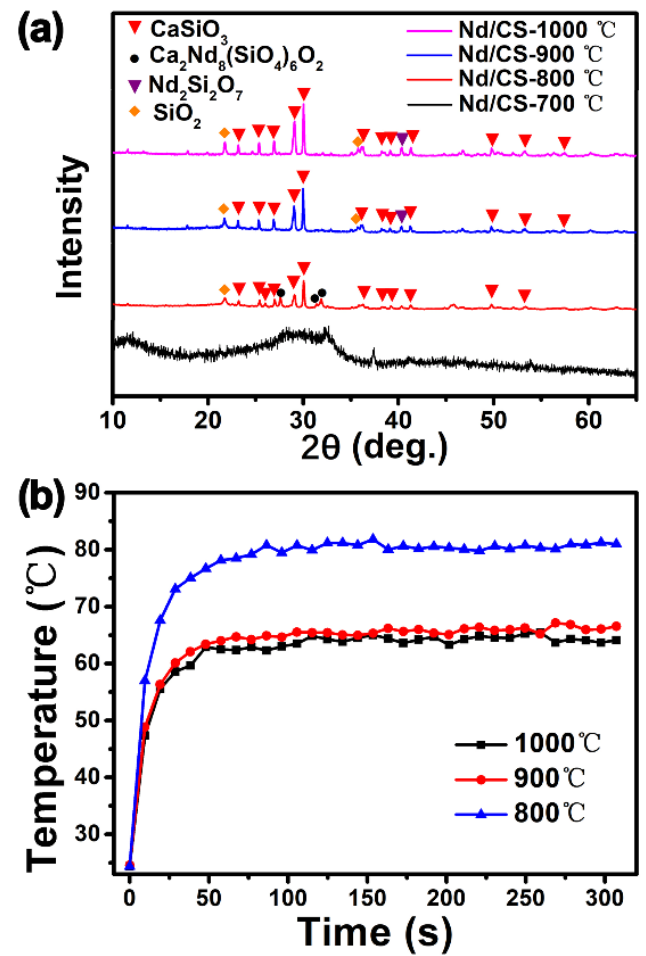

图 1 不同温度烧结所得 $\mathrm{Nd} / \mathrm{CS}$ 生物陶瓷粉体的物相组成与 光热性能表征

Fig. 1 Phase composition and photothermal property of Nd/CS bioceramic powders after sintering at different temperatures

(a) XRD patterns of the $\mathrm{Nd} / \mathrm{CS}$ bioceramic powders after sintering at different temperatures. (b) Photothermal performance $\left(808 \mathrm{~nm}\right.$ laser, $0.6 \mathrm{~W} / \mathrm{cm}^{2}$ ) of $\mathrm{Nd} / \mathrm{CS}$ bioceramic powders with different sintering temperature

图 2 展示了 $800{ }^{\circ} \mathrm{C}$ 烧结后 $\mathrm{Nd} / \mathrm{CS}$ 陶瓷粉体的 光热性能以及光热稳定性。未引入 $\mathrm{Nd}$ 离子的硅酸 钲没有光热性能, 而引入 $\mathrm{Nd}$ 离子的 $\mathrm{Nd} / \mathrm{CS}$ 粉体具 有优异的光热性能 (图 2(a))。在 $808 \mathrm{~nm}$ 近红外、 $0.5 \mathrm{~W} / \mathrm{cm}^{2}$ 功率激光照射下, 陶瓷粉体的温度快速升 高达到一定数值并保持稳定温度, 并且随着激光功 率的增加, $\mathrm{Nd} / \mathrm{CS}$ 粉体受激光照射后的温度也随之 增加 (图 2(b))。这说明调节激光功率可以进一步调 节粉体的光热性能。此外, 如图 2(c)所示, 通过 4 次 激光的开/关循环检测, $\mathrm{Nd} / \mathrm{CS}$ 陶瓷粉体都能达到相 同的温度值, 表明 $\mathrm{Nd} / \mathrm{CS}$ 生物陶瓷粉体具有优异的 
光热稳定性。

(a)

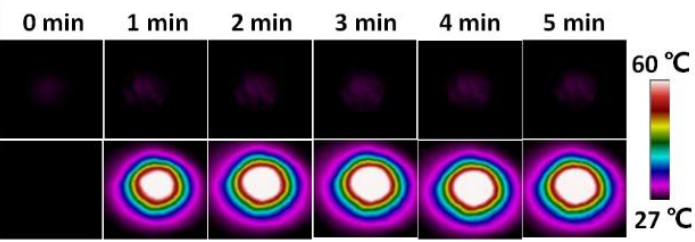

(b)

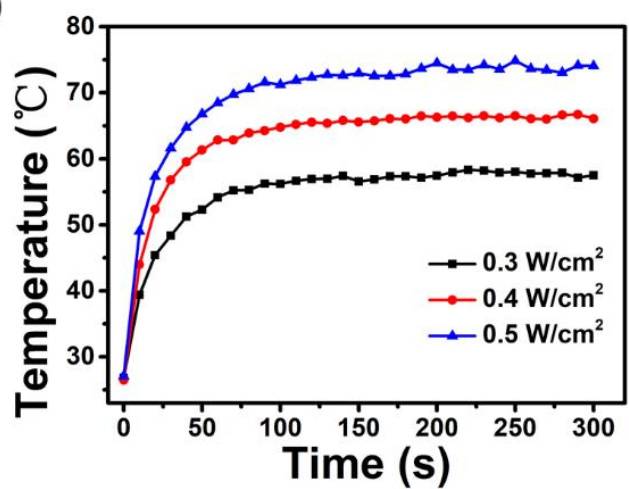

(c)

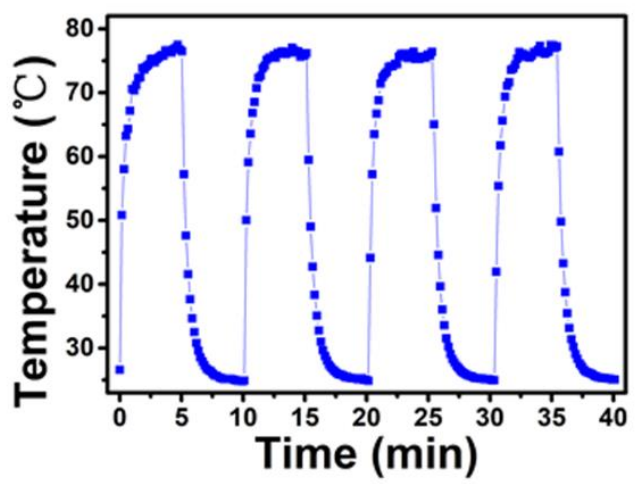

图 $2 \mathrm{Nd} / \mathrm{CS}$ 生物陶瓷粉体的光热性能

Fig. 2 Photothermal properties of Nd/CS bioceramic powder

(a) Photothermal images of the powder at different moments under 0.5 $\mathrm{W} / \mathrm{cm}^{2}$ power density. (b) Photothermal curve of powder under different laser power densities. (c) Powder stability under the photothermal conditions of $808 \mathrm{~nm}$ laser and $0.5 \mathrm{~W} / \mathrm{cm}^{2}$

\section{$2.2 \mathrm{Nd} / \mathrm{CS}$ 生物陶瓷粉体的荧光性能}

$\mathrm{Nd} / \mathrm{CS}$ 陶瓷粉体的荧光性能表征结果如图 3 所 示。 $\mathrm{Nd} / \mathrm{CS}$ 陶瓷粉体在 $808 \mathrm{~nm}$ 的激光照射下，显示 出三个主要的 $\mathrm{Nd}$ 离子电子跃迁发射光谱的特征苂 光光谱 ${ }^{[5]}$ : $884 、 1062$ 和 $1336 \mathrm{~nm}$ 附近(图 3(a)), 分 别对应 $\mathrm{Nd}$ 离子的 ${ }^{4} \mathrm{~F}_{3 / 2} \rightarrow{ }^{4} \mathrm{~F}_{9 / 2},{ }^{4} \mathrm{~F}_{3 / 2} \rightarrow{ }^{4} \mathrm{~F}_{11 / 2},{ }^{4} \mathrm{~F}_{3 / 2} \rightarrow$ ${ }^{4} \mathrm{~F}_{13 / 2}$ 跃迁。但是与 $\mathrm{Nd}$ 离子特征光谱相比, $\mathrm{Nd} / \mathrm{CS}$ 陶瓷粉体的光谱跃迁分离出了多组谱峰, 分别在 $\mathrm{Nd}$ 离子的特征苂光光谱附近： $884 \mathrm{~nm} \mathrm{（870} \mathrm{nm、878}$ $\mathrm{nm} 、 884 \mathrm{~nm} 、 898 \mathrm{~nm} 、 914 \mathrm{~nm} 、 926 \mathrm{~nm}), 1062 \mathrm{~nm}$ ( $1057 \mathrm{~nm} 、 1063 \mathrm{~nm} 、 1083 \mathrm{~nm} 、 1096 \mathrm{~nm}), 1336 \mathrm{~nm}$ $(1328 \mathrm{~nm} 、 1336 \mathrm{~nm})$ 。这主要是由于在硅酸钙中引 入 $\mathrm{Nd}$ 离子后, $\mathrm{Nd}^{3+}$ 周围不同的晶体场环境造成的 [18-19]。另外, $\mathrm{Nd} / \mathrm{CS}$ 陶瓷粉体的苂光强度随着激光 功率的增加而有规律地增加（图 3(b)）。值得注意的
是 $\mathrm{Nd} / \mathrm{CS}$ 陶瓷粉体在 $1063 \mathrm{~nm}$ 处的苂光强度与温度 呈线性关系 (图 3(c))。这种有规律的线性对应关系， 有望在生物医学领域用于对生物体的非侵入式温度 监测 $[6,20-21]$ 。

(a)

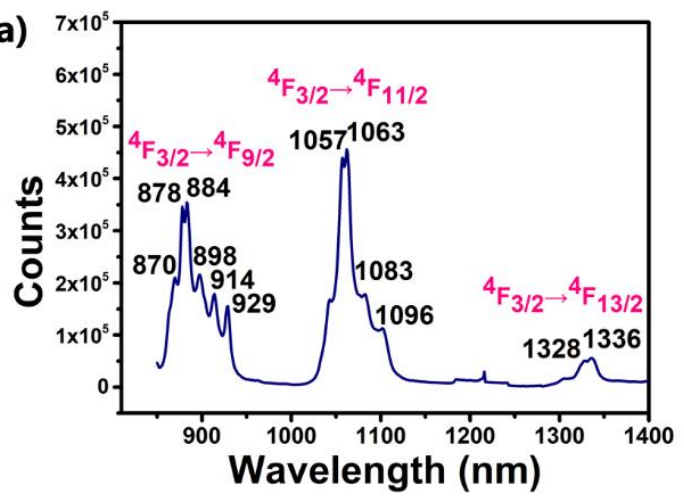

(b)

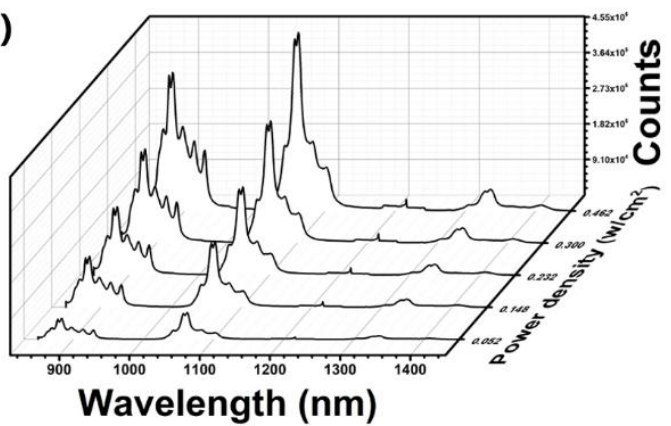

(c)

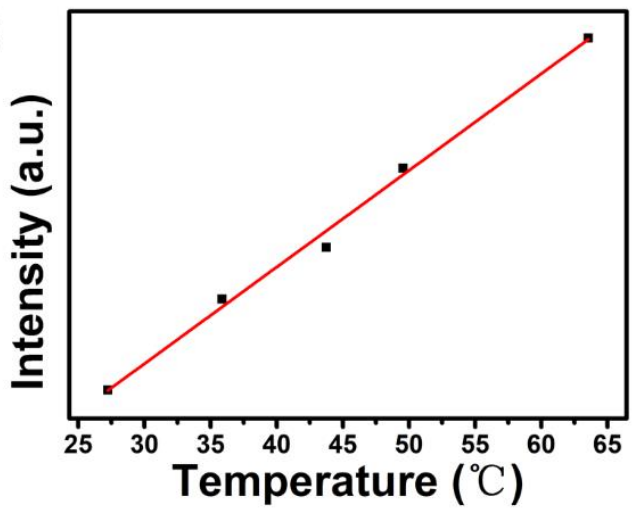

图 $3 \mathrm{Nd} / \mathrm{CS}$ 陶瓷粉体的苂光性能

Fig. 3 Fluorescence properties of Nd/CS ceramic powder (a) Fluorescence emission spectrum of the powder at room temperature under $0.462 \mathrm{~W} / \mathrm{cm}^{2}$ laser power density; (b) Fluorescence intensity under different laser power densities; (c) Linear fitting relationship between $1063 \mathrm{~nm}$ fluorescence intensity and temperature for $\mathrm{Nd} / \mathrm{CS}$ powders

$2.3 \mathrm{Nd} / \mathrm{CS}-\mathrm{PCL}$ 静电纺丝复合膜的微观形貌、光 热性能与荧光性能

为了使多功能 $\mathrm{Nd} / \mathrm{CS}$ 陶瓷粉体应用于软组织损 伤修复，本实验通过静电纺丝技术将 $\mathrm{Nd} / \mathrm{CS}$ 陶瓷粉 体与 PCL 复合制备成静电纺丝复合膜（图 4(a)）。 低倍扫描电镜照片显示, 加入 $\mathrm{Nd} / \mathrm{CS}$ 陶瓷粉体后, 复合膜的纤维结构形貌没有明显变化。高倍扫描电 
镜照片显示，加入陶瓷粉体后复合膜的纤维丝表面 没有颗粒状的粉体，说明 $\mathrm{Nd} / \mathrm{CS}$ 陶瓷粉体都被包裹 在纤维丝内部。虽然高倍照片局部的纤维丝直径存 在差异, 但陶瓷粉体的加入并没有明显改变纤维丝 的直径大小。纤维丝直径的定量分析也显示, 加入 $\mathrm{Nd} / \mathrm{CS}$ 陶瓷粉体前后静电纺丝的直径都在 $0.3 \sim 3.7$ $\mu \mathrm{m}$ 之间,证实陶瓷粉体的加入并没有明显改变纤维 丝的直径大小。对静电纺丝复合膜进行光热性能表 征显示 (图 4(b)), 激光功率密度在 $2 \mathrm{~W} / \mathrm{cm}^{2}$ 时, $\mathrm{Nd} / \mathrm{CS}-\mathrm{PCL} 10$ 静电纺丝复合膜快速升温达到 $54^{\circ} \mathrm{C}$ 并保持温度恒定。而 PCL 与 Nd/CS-PCL5 复合膜的 温度虽有一定的上升，但却达不到生物光热治疗的 温度。这些结果表明制备的静电纺丝复合膜依然保 持着 $\mathrm{Nd} / \mathrm{CS}$ 陶瓷粉体的光热性能, 并可通过调节复 合电纺丝中复合的 $\mathrm{Nd} / \mathrm{CS}$ 陶瓷粉体含量与激光功率 进一步调节静电纺丝复合膜的光热性能。

$\mathrm{Nd} / \mathrm{CS}-\mathrm{PCL} 10$ 静电纺丝复合膜的荧光性能表征 结果 (图 4(c,d) ) 显示, $\mathrm{Nd} / \mathrm{CS}$ 粉体与 PCL 复合制 备成纺丝膜后, 在 $808 \mathrm{~nm}$ 的激光作用下仍然具有与 $\mathrm{Nd} / \mathrm{CS}$ 粉体类似的良好荧光性能。且随着激光功率 的增加, 静电纺丝复合膜的荧光强度随之呈线性增 加。在 $1063 \mathrm{~nm}$ 处，静电纺丝复合膜的苂光强度与 温度依旧呈线性关系。说明静电纺丝复合膜具有温 度检测的功能。

\section{$2.4 \mathrm{Nd} / \mathrm{CS}-\mathrm{PCL}$ 静电纺丝复合膜活性离子释放}

前期研究表明 $\mathrm{Ca} 、 \mathrm{Si}$ 离子在组织修复过程中发 挥着重要作用 $[14,22]$ 。因此, 本研究对静电纺丝复合
膜释放 $\mathrm{Ca} 、 \mathrm{Si}$ 离子的能力作了检测。图 4(e,f) 展示 了复合膜在 $\mathrm{PBS}$ 中 $\mathrm{Ca} 、 \mathrm{Si}$ 离子的释放能力。随着 电纺丝膜浸泡时间的延长, 复合膜的 $\mathrm{Ca} 、 \mathrm{Si}$ 离子释 放量也随之增加，且在 $1 、 3 、 5$ 和 $7 \mathrm{~d}$ 期间复合膜 的离子累积释放量逐渐增加。这表明复合膜具有缓 释离子的效果。此外, $\mathrm{Si}$ 离子释放量在能够促进皮 肤成血管、增加成骨细胞活性的离子浓度范围之内 [23]，具有促进组织损伤修复的潜力。

\section{$2.5 \mathrm{Nd} / \mathrm{CS}-\mathrm{PCL}$ 静电纺丝复合膜的生物学性能}

为了研究静电纺丝复合膜的生物学性能, 本实 验研究了 Nd/CS-PCL 复合膜对 HDF 细胞迁移、粘 附的影响 (图 5 )。与纯 PCL 静电纺丝膜相比, Nd/CSPCL5 与 Nd/CS-PCL10 静电纺丝复合膜均具有一定 的促进细胞迁移的作用 (图 5(a)), 定量分析结果也 表明, Nd/CS-PCL 静电纺丝复合膜确实可以明显促 进 HDF 细胞迁移（图 5(b)）。 HDF 细胞均可粘附在 复合进纺丝膜上, 铺展良好 (图 5(c))。从细胞形态 可以看出, $\mathrm{Nd} / \mathrm{CS}-\mathrm{PCL} 5$ 与 $\mathrm{Nd} / \mathrm{CS}-\mathrm{PCL} 10$ 静电纺丝 复合膜上的细胞更容易进入复合膜孔内，具有更优 异的细胞迁移、粘附作用。这些细胞实验结果说明, 静电纺丝复合膜具有优良的生物学性能, 这可能是 由于释放活性 $\mathrm{Si}$ 离子（硅酸根离子）促进了 $\mathrm{HDF}$ 细胞的迁移、粘附 [24]。不过，为了进一步证明静电 纺丝复合膜在组织损伤修复方面的应用, 后续还需 作深入的生物学评价, 包括细胞成血管基因表达及 体内动物创伤模型实验验证等。
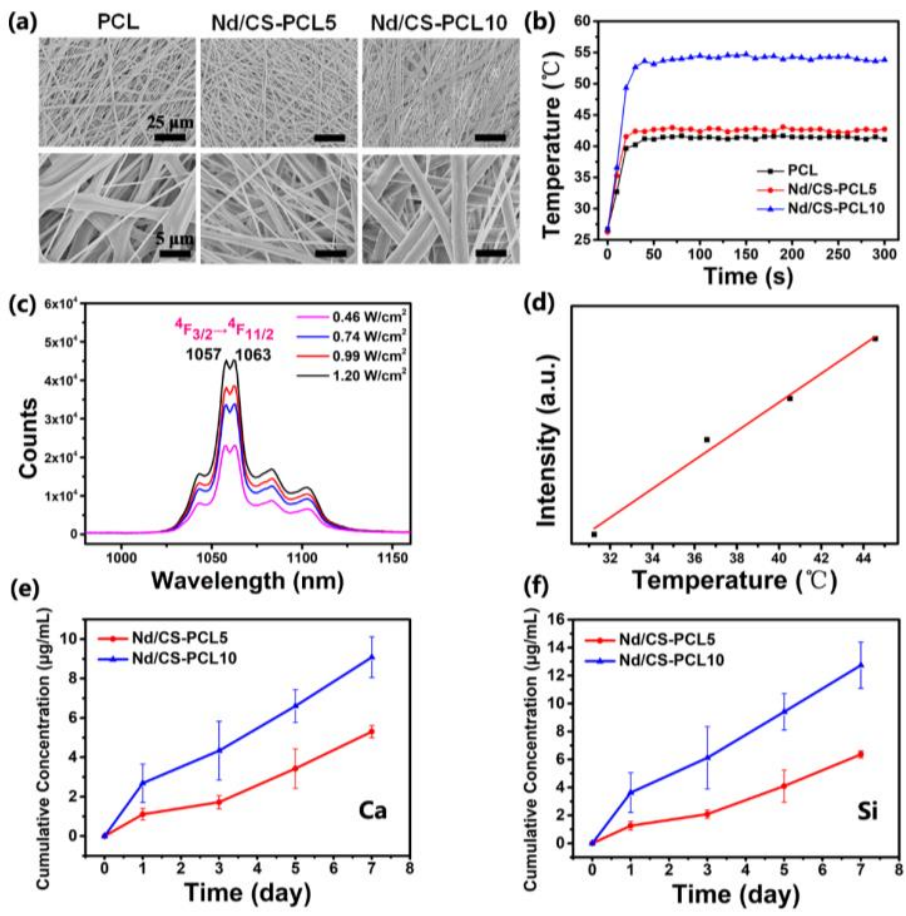
图 4 静电纺丝复合膜的微观形貌、光热性能、苂光性能及离子释放

Fig. 4 Microscopic morphology, photothermal performance, fluorescence performance, and ion release property of composite electrospun membrane

(a) SEM images of the composite electrospun membrane; (b) Photothermal curves of the electrospun membrane at a laser power density of 2 W/cm ${ }^{2}$; (c) Fluorescence intensity of the composite electrospun membrane under different laser power densities (1057 nm and $1063 \mathrm{~nm}$ ); (d) Linear relationship between temperature and fluorescence intensity $(1063 \mathrm{~nm}$ ) for the composite electrospun membrane. (e, f) Amount of Ca ions (e) and $\mathrm{Si}$ ions (f) released from the composite membrane after $1,3,5$, and $7 \mathrm{~d}$
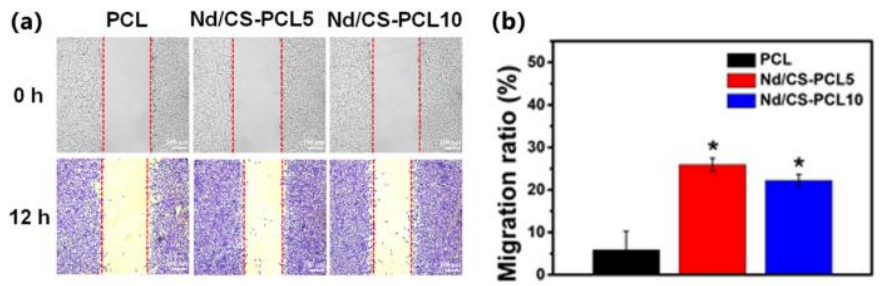

(c)
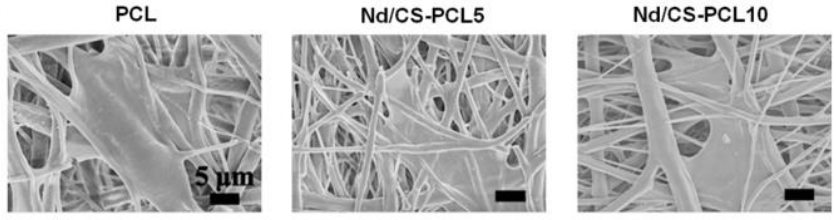

图 5 复合电纺丝膜的生物学性能

Fig. 5 Biological properties of composite electrospun membrane

(a) Photographs of HDF cell migration at 0 and $12 \mathrm{~h}$ after scratching; (b) Quantitative expression of cell migration at $12 \mathrm{~h}$; (c) SEM images of cell adhesion and in-growth of HDF cells after $24 \mathrm{~h}$ of culture

\section{3 结论}

对 $\mathrm{Nd}-\mathrm{Ca}-\mathrm{Si}$ 生物陶瓷粉体及复合电纺丝膜的理 化性能及生物性能的研究发现:

1) 采用化学共沉淀法成功制备出了以硅酸钻为主 相、含有 $\mathrm{Ca}_{2} \mathrm{Nd}_{8}\left(\mathrm{SiO}_{4}\right)_{6} \mathrm{O}_{2}$ 和 $\mathrm{Nd}_{2} \mathrm{Si}_{2} \mathrm{O}_{7}$ 相的 $\mathrm{Nd}-$ $\mathrm{Ca}-\mathrm{Si}$ 生物活性陶瓷。

2) Nd-Ca-Si 陶瓷粉体具有光热、苂光多功能性能。 其中含 $\mathrm{Ca}_{2} \mathrm{Nd}_{8}\left(\mathrm{SiO}_{4}\right)_{6} \mathrm{O}_{2}$ 相的陶瓷光热性能更好。

3) 通过静电纺丝技术可以制备出陶瓷/PCL复合膜, 且复合膜具有良好的光热性能及生物学性能。通 过调节激光功率密度及 $\mathrm{Nd} / \mathrm{CS}$ 粉体的复合量, 可 进一步调节静电纺丝复合材料的光热性能。

研究结果显示, Nd-Ca-Si 生物陶瓷粉体、陶瓷粉 体/高分子静电纺丝复合膜具有光热、苂光发光性能 且苂光强度与温度成线性关系。同时, 陶瓷复合纤维 膜还具有良好的生物活性。因此, Nd-Ca-Si 生物陶瓷 及其复合材料可望应用于肿瘤光热治疗、荧光成像/ 测温、促进生物组织修复等方面。

\section{参考文献:}

[1] ZU B B, ZHANG W, ZHANG Z J, et al. Photothermal enhanced photocatalytic properties of itanium dioxide (B)/glass fiber cloth. Journal of Inorganic Materials, 2019, 34(9): 961-966.

[2] XIE X, WU J R, CAI X J, et al. Photothermal/pH response B-CuSDOX nanodrugs for chemo-photothermal synergistic therapy of tumor.
Journal of Inorganic Materials, 2021, 36(1): 81-87.

[3] ZENG Y L, CHEN J J, TIAN Z F, et al. Preparation of mesoporous organosilica-based nanosystem for in vitro synergistic chemo-and photothermal therapy. Journal of Inorganic Materials, 2020, 35(12): $1-8$.

[4] ZHAO L, LIU Y, XING R, et al. Supramolecular photothermal effects: a promising mechanism for efficient thermal conversion. Angew. Chem. Int. Ed. Engl., 2020, 59(10): 3793-3801.

[5] DEL R B, PéREZ-DELGADO A, MISIAK M, et al. Neodymiumdoped nanoparticles for infrared fluorescence bioimaging: the role of the host. Journal of Applied Physics, 2015, 118(14): 143104.

[6] CARRASCO E, DEL R B, SANZ-RODRíGUEZ F, et al. Intratumoral thermal reading during photo-thermal therapy by multifunctional fluorescent nanoparticles. Advanced Functional Materials, 2015, 25(4): 615-626.

[7] ZHONG J, CHEN D, PENG Y, et al. A review on nanostructured glass ceramics for promising application in optical thermometry. Journal of Alloys and Compounds, 2018, 763: 34-48.

[8] GNACH A, LIPINSKI T, BEDNARKIEWICZ A, et al. Upconverting nanoparticles: assessing the toxicity. Chemical Society Reviews, 2015, 44(6): 1561-1584.

[9] MARGRIET V P, Danielle P L, HENK V L, et al. The status of in vitro toxicity studies in the risk asssessment of nanomaterials. Nanomedicine, 2009, 4(6): 660-685.

[10] LV R, YANG G, HE F, et al. $\mathrm{LaF}_{3}: \mathrm{Ln}$ mesoporous spheres: controllable synthesis, tunable luminescence and application for dualmodal chemo-/photo-thermal therapy. Nanoscale, 2014, 6(24): 1479914809 .

[11] WU S, BUTT H-J. Near-infrared-sensitive materials based on upconverting nanoparticles. Advanced Materials, 2016, 28(6): 12081226.

[12] HAASE M, SCHAEFER H. Upconverting nanoparticles. Angewandte Chemie-International Edition, 2011, 50(26): 5808-5829.

[13] WANG C, LIN K, CHANG J, et al. Osteogenesis and angiogenesis induced by porous beta- $\mathrm{CaSiO}_{3} / \mathrm{PDLGA}$ composite scaffold via activation of AMPK/ERK1/2 and PI3K/Akt pathways. Biomaterials, 2013, 34(1): 64-77.

[14] LI H, CHANG J. Stimulation of proangiogenesis by calcium silicate bioactive ceramic. Acta Biomater., 2013, 9(2): 5379-5389.

[15] ZHAI W, LU H, CHEN L, et al. Silicate bioceramics induce angiogenesis during bone regeneration. Acta Biomater., 2012, 8(1): 341349 .

[16] WU C T, CHANG J. Silicate bioceramics for bone tissue 
regeneration. Journal of Inorganic Materials, 2013, 28(1): 29-39.

[17] YU Q, CHANG J, WU C. Silicate bioceramics: from soft tissue regeneration to tumor therapy. J. Mater. Chem. B, 2019, 7(36): 54495460.

[18] CRUM J V, CHONG S, PETERSON J A, et al. Syntheses, crystal structures, and comparisons of rare-earth oxyapatites $\mathrm{Ca}_{2} \mathrm{RE}_{8}\left(\mathrm{SiO}_{4}\right)_{6} \mathrm{O}_{2}$ ( $\mathrm{RE}=\mathrm{La}, \mathrm{Nd}, \mathrm{Sm}, \mathrm{Eu}$, or $\mathrm{Yb}$ ) and $\mathrm{NaLa}_{9}\left(\mathrm{SiO}_{4}\right)_{6} \mathrm{O}_{2}$. Acta Crystallogr $E$ Crystallogr Commun, 2019, 75: 1020-1025.

[19] PENG F, ZHANG Q L, WANG X F, et al. Synthesis, structure and spectroscopic properties of $\mathrm{Nd}^{3+}: \mathrm{SrY}_{2} \mathrm{O}_{4}$ phosphor. Journal of Physics, 2016, 65(1): 1-6.

[20] FIORENZO V, RAFIF N, ALICIA Z, et al. Temperature sensing using fluorescent nanothermometers. ACS Nano, 2010, 4(6): 3254-3258.

[21] JAQUE D, JACINTO C. Luminescent nanoprobes for thermal bio-sensing: towards controlled photo-thermal therapies. Journal of Luminescence, 2016, 169:394-399.

[22] WU C T, FAN W, ZHOU Y H, et al. 3D-printing of highly uniform $\mathrm{CaSiO}_{3}$ ceramic scaffolds: preparation, characterization and in vivo osteogenesis. J Mater. Chem., 2012, 22: 12288-12295.

[23] HOPPE A, GULDAL N S, BOCCACCINI A R. A review of the biological response to ionic dissolution products from bioactive glasses and glass-ceramics. Biomaterials, 2011, 32(11): 2757-2774.

[24] LI H, CHANG J. Bioactive silicate materials stimulate angiogenesis in fibroblast and endothelial cell co-culture system through paracrine effect. Acta Biomater., 2013, 9(6): 6981-6991. 